\title{
Descriptive epidemiology of high frequency component based on heart rate variability from 10-second ECG data and daily physical activity among community adult residents: the Nagahama Study
}

\author{
Naomi Takahashi, ${ }^{1, *}$, Yoshimitsu Takahashi ${ }^{1}$, Yasuharu Tabara ${ }^{2}$, Takahisa Kawaguchi ${ }^{2}$, \\ Akira Kuriyama $^{1}$, Kenji Ueshima ${ }^{3}$, Shinji Kosugi ${ }^{4}$, Akihiro Sekine ${ }^{5}$, Ryo Yamada ${ }^{2}$, \\ Fumihiko Matsuda ${ }^{2}$, Takeo Nakayama ${ }^{1}$, On behalf of the Nagahama Study Group \\ ${ }^{1}$ Department of Health Informatics, Kyoto University School of Public Health, Kyoto, Japan; \\ ${ }^{2}$ Center for Genomic Medicine, Graduate School of Medicine, Kyoto University, Kyoto, Japan; \\ ${ }^{3}$ Department of EBM Research, Institute for Advancement of Clinical and Translational Science, Kyoto University Hospital, Kyoto, Japan; \\ ${ }^{4}$ Department of Medical Ethics and Medical Genetics, Kyoto University School of Public Health, Kyoto, Japan; \\ ${ }^{5}$ Department of Omics-based Medicine, Clinical Preventive Medical Sciences, Center for Preventive Medical Sciences, Chiba University, Chiba, \\ Japan.
}

SUMMARY Characteristics of high frequency (HF) component based on heart rate variability (HRV) in a large general population remain unclear, particularly on the relationship with daily physical activity. We aimed to characterize the distribution of HF component and examine the association with daily physical activity among community residents. We performed spectral analysis of HRV from 10-second ECG recordings among 9135 residents aged 30 to 74 years in Nagahama City, Japan. HF components were log-transformed to consider the distribution. Simple correlations between HF and age were determined. Age-adjusted mean values of HF component were calculated for each questionnaire item related to daily physical activity. Multiple regression analysis was performed to examine the effect of daily physical activity on HF component value. Mean values of logarithmicallytransformed HF component (lnHF) were higher in women than in men $(p<0.001)$. lnHF was inversely associated with age ( $r=-0.40,-0.49$ for men, women, respectively). Adjusted mean $\operatorname{lnHF}$ for physically active people was significantly higher than that in inactive people $(p<0.001)$. HF components from 10-second ECG recordings were moderately and negatively correlated with age in both sexes, and positively correlated with daily physical activity in the general adult population. Maintaining the level of daily physical activity, especially to exercise regularly could keep the parasympathetic function high.

Keywords parasympathetic function, spectral analysis, short ECG recordings

\section{Introduction}

One way to evaluate autonomic nervous system function is to analyze heart rate variability (HRV), a parameter that can offer a pathophysiologic perspective on cardiovascular disease (CVD) including coronary artery disease, heart failure, and arrhythmias $(1,2)$. In particular, spectral analysis of HRV using the Fast Fourier Transform (FFT) $(3,4)$, the autoregressive model (AR model) (4,5), or the Maximum Entropy Method (MEM) (4) allows for the separate evaluation of sympathetic and parasympathetic nervous activity. These methods are all based on the fact that sympathetic and parasympathetic nerves reflect HRV of specific frequency bands $(1,2)$. High-frequency (HF) component $(0.15-0.4 \mathrm{~Hz})$ is an index of the parasympathetic nerve function, which is subject to the respiration. Low-frequency (LF) component $(0.04-0.15 \mathrm{~Hz})$ is influenced by both of parasympathetic and sympathetic nerve activity, and thus the ratio of LF to $\mathrm{HF}$ (LF/HF) is considered as an index of the sympathetic nerve function. Some studies have examined the relationships between HRV and various diseases, and have found that decreased parasympathetic function is associated with coronary heart disease (CHD) risk and mental stress $(6,7)$. However, the distribution and characteristics of the HF and LF components have not been fully elucidated in a general population. When electrocardiogram (ECG) is used for general evaluations of parameters such as autonomic nervous function, a basic ECG is run for 5 minutes $(2,8)$. Some large cohort 
studies had their findings based on analyzed HRV from ECG recordings shorter than 5 minutes $(7,9)$. The results of HRV analysis from shorter ECG recordings comparing with longer recordings was previously validated $(10,11)$.

It is well known that exercise can positively affect health, and the presence or absence of exercise habits has greatly impacted the health maintenance of the human mind and body $(12,13)$. Physical activity such as exercise can have soothing effects on the autonomic nervous system $(14,15)$. Some studies have found an association between certain lifestyle habits and HRV in large-scale studies of community residents, but the association between daily physical activity habits and HRV has not been fully elucidated (16-18).

In Japan, mass health checkups for adults are typically conducted in community settings, and where an ECG is to be recorded for only 10 seconds. We previously validated 10 -second recording of ECG to estimate HF component comparing with 5-minute recording (19). It became possible to estimate HF component from routine short ECG recording in mass surveys, and large number of ECG data would be available for this purpose.

We hypothesized that the HF component values obtained from 10-second ECG data were reflective of exercise habits. However, the little evidence is available to suggest a relationship between HF component analyzed from short ECG recordings and physical activity. The present study aimed to estimate the HF component on the 10-second ECG and examine the association between daily physical activity and HF component in a large community population.

\section{Methods}

\subsection{Study design and study population}

A cross-sectional study was conducted using baseline data from the Nagahama Prospective Genome Cohort for Comprehensive Human Bioscience (The Nagahama Study (20)). This cohort comprising healthy community residents (aged 30 to 74 years) of Nagahama City (population, 125,000), Shiga Prefecture, located in the center of Japan, was recruited from 2008 to 2010. Out of the eligible resident, 10,082 people who agreed to participate after receiving the explanation of a community-based genome-epidemiologic study, the "Nagahama Zero (0)-ji Prevention Cohort Project" were selected as participants in the Nagahama Study. We had recruited participants of health checkups from about 70,000 eligible people by through public relations activities with the Nagahama city and nonprofit organization Zeroji Club, and participating in voluntary. Of the 10,082 participants, we excluded 278 people for the following reasons: participants in the pilot study ( $n=273$; due to the improvement of the health checkup contents for this survey based on

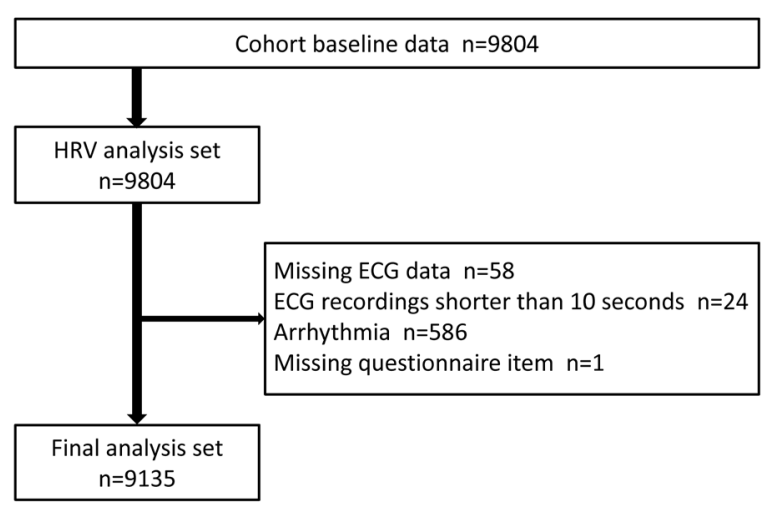

Figure 1. Flowchart of study subject selection.

the pilot's implementation, missing questionnaire $(n=$ 2), missing almost blood and physiological tests $(n=$ 3). Finally, 9,804 participants were confirmed as the Nagahama Study 1st phase cohort data. We applied our study inclusion criteria to this 1 st phase cohort data. Of the 9,804 participants, we excluded certain individuals due to missing ECG data, ECG recordings shorter than 10 seconds, presence of an arrhythmia and missing necessary questionnaire item (Figure 1). Inappropriate arrhythmia types for spectral analysis were determined according to the Minnesota code $(21,22)$ through discussion among co-authors (NT: registered nurse, AK \& TN: general physician, KU: cardiologist). The study protocol was approved by Kyoto University Graduate School and Faculty of Medicine, Ethics Committee (E1495 and G278).

\subsection{Electrocardiogram and spectral analysis of $\mathrm{HRV}$}

Standard 12-lead electrocardiograms (FCP-7431, Fukuda Denshi, Tokyo, Japan) were recorded for 10 seconds in the supine position in the baseline survey of The Nagahama Study. ECG recordings in our study were performed between 9:00 a.m. and 4:00 p.m.

We used the MemCalc method in this study, so we used software MemCalc/Win (Suwa Trust, Tokyo, Japan) for HRV analysis. This method is a type of time-series data analysis using the Maximum Entropy Method (MEM) for spectral analysis of the frequency domain, and the nonlinear least squares model for analysis of the time domain (reproduction of the time series) to determine underlying variation in the time series $(5,23,24)$. As the ECG data length required in the frequency band was calculated as $1 /$ frequency (seconds), the minimum data lengths required for analysis of HF and LF components were 6.7 and 25 seconds, respectively. As our ECG data from the baseline study of the Nagahama Study were from 10-second ECGs, we were only able to evaluate the HF component.

\subsection{Daily physical activity habits}

From the baseline questionnaire, the following three 
items were used for the present purpose: "Are you in the habit of exercising to the point of light sweating for over 30 minutes per session, twice weekly, for over a year?" ("Yes" or "No"); "In your daily life do you walk or do an equivalent amount of physical activity for more than one hour a day?" ("Yes" or "No"); and "How would you describe your physical activity level in your daily life?" ("Insufficiently active", "Sufficiently active", or "Highly active")

\subsection{Statistical analysis}

Descriptive statistics of participants' characteristics (age, height, weight, body mass index (BMI), blood pressure, and brain natriuretic peptide (BNP)), HF component, R-R interval, and heart rate from R-R interval were described. The $t$-test and Wilcoxon rank sum test to compare continuous values, and chi-square test to compare categorical values were used. Pearson's correlation analysis was conducted to examine the association between age and HF components. The normality of crude values of the HF component was examined by histograms, box plots, normal probability plots, and the Shapiro-Wilk test. Pearson's correlation coefficients were calculated between age and HF component values, systolic blood pressure (SBP), and diastolic blood pressure (DBP). For the HF component, analysis of covariance for each questionnaire item related to physical activity was carried out, and means were calculated for the HF component value after adjusting for age. In addition, in order to examine the effect of physical activity on the HF component value, three multiple regression models were created with logarithmicallytransformed values of HF component as the response variable and physical activity habits, age, sex, and BMI as explanatory variables (Model A: age, sex, BMI, and exercise to the point of sweating; Model B: age, sex, $\mathrm{BMI}$, and physical activity $>1 \mathrm{~h}$; Model C: age, sex, BMI, and physical activity level). All statistical analyses were performed with Stata SE Ver.13.1 (College Station, TX). Statistical significance tests were two-sided and $P<$ 0.05 was considered significant.

\section{Results}

\subsection{Selection of participants for analysis}

Of the 9,804 participants, we excluded 669 people for the following reasons: missing ECG data $(n=58)$, ECG recordings shorter than 10 seconds $(n=24)$, presence of an arrhythmia $(n=586)$, and missing necessary questionnaire item $(n=1)$ (Figure 1$)$.

\subsection{Participant characteristics}

Table 1 shows characteristics of the 9,135 participants selected for analysis and the results of the HRV analysis. Mean participant ages were 55.4 and 52.4 years for men and women, respectively, with relatively few participants in their $40 \mathrm{~s}(14 \%)$ and $70 \mathrm{~s}(11 \%)$. As normality of the HF component was not evident, logarithmic transformation was applied for analysis. The mean logarithmicallytransformed HF component (lnHF) value for women (5.34) was higher than that for men (5.11) $(p<0.001)$.

Table 2 shows means of $\operatorname{lnHF}$ values for each age group among those in their 30 s to 50 s, women had a higher mean HF component value than men, while among those in their 60 s to $70 \mathrm{~s}$, the men exhibited a higher mean HF component value than women. Mean lnHF values decreased with increases in age group. The

Table 1. Participant characteristics and heart rate variability from 10-second ECG data

\begin{tabular}{|c|c|c|c|c|}
\hline & Total $(n=9,135)$ & $\operatorname{Men}(n=2,932)$ & Women $(n=6,203)$ & $P$ value \\
\hline Age group & & & & $<0.001$ \\
\hline $30-39$ years & $2167(24 \%)$ & $611(21 \%)$ & $1556(25 \%)$ & \\
\hline 40-49 years & $1313(14 \%)$ & $360(12 \%)$ & $953(15 \%)$ & \\
\hline $50-59$ years & $1811(20 \%)$ & $456(16 \%)$ & $1355(22 \%)$ & \\
\hline $60-69$ years & $2885(32 \%)$ & $1091(37 \%)$ & $1794(29 \%)$ & \\
\hline $70-79$ years & $959(11 \%)$ & $414(14 \%)$ & $545(9 \%)$ & \\
\hline Age (years) & $53.3 \pm 13.3$ & $55.4 \pm 13.5$ & $52.4 \pm 13.1$ & $<0.001$ \\
\hline Height $(\mathrm{cm})$ & $159.9 \pm 8.4$ & $168.3 \pm 6.5$ & $155.9 \pm 5.9$ & $<0.001$ \\
\hline Weight (kg) & $57.2 \pm 10.9$ & $66.5 \pm 10.2$ & $52.9 \pm 8.1$ & $<0.001$ \\
\hline BMI $\left(\mathrm{kg} / \mathrm{m}^{2}\right)$ & $22.3 \pm 3.3$ & $23.4 \pm 3.1$ & $21.7 \pm 3.2$ & $<0.001$ \\
\hline \multicolumn{5}{|c|}{ Blood pressure (mm Hg) } \\
\hline SBP & $121.8 \pm 17.0$ & $128.5 \pm 15.7$ & $118.7 \pm 16.7$ & $<0.001$ \\
\hline DBP & $75.3 \pm 11.1$ & $79.9 \pm 10.8$ & $73.2 \pm 10.6$ & $<0.001$ \\
\hline $\mathrm{BNP}(\mathrm{pg} / \mathrm{mL})$ & $12.3[7.3,20.2]$ & $10.1[5.9,18]$ & $13.1[8.1,21.4]$ & $<0.001$ \\
\hline Heart rate(bpm) & $64[58,70]$ & $62[57,68]$ & $64[59,70]$ & $<0.001$ \\
\hline $\mathrm{R}-\mathrm{R}$ (ms) & $946.5 \pm 127.7$ & $968.5 \pm 136.7$ & $936.1 \pm 121.8$ & $<0.001$ \\
\hline $\ln \mathrm{HF}$ & $5.26 \pm 1.48$ & $5.11 \pm 1.47$ & $5.34 \pm 1.48$ & $<0.001$ \\
\hline
\end{tabular}

SBP: systolic blood pressure; DBP: diastolic blood pressure; BNP: brain natriuretic peptide; R-R: mean variation in R-R interval; lnHF: logtransformed HF component values; Mean \pm SD: Age, Height, Weight, BMI, Blood pressure, R-R, lnHF. Median [first quartile, third quartile]: BNP, Heart rate. Comparisons between men and women: $P$-value for age group, chi-square test; $P$-value for mean, $t$-test; $P$-value for median, Wilcoxon rank sum test. 
Table 2. Mean \pm SD of InHF from 10-second ECG data

\begin{tabular}{lccc}
\hline Age (years) & Total $(n=9,135)$ & Men $(n=2,932)$ & Women $(n=6,203)$ \\
\hline $30-39$ & $6.30 \pm 1.19$ & $6.11 \pm 1.23$ & $6.38 \pm 1.16$ \\
$40-49$ & $5.75 \pm 1.26$ & $5.53 \pm 1.35$ & $5.83 \pm 1.21$ \\
$50-59$ & $5.02 \pm 1.26$ & $4.94 \pm 1.31$ & $5.05 \pm 1.25$ \\
$60-69$ & $4.68 \pm 1.38$ & $4.71 \pm 1.39$ & $4.66 \pm 1.38$ \\
$70-79$ & $4.46 \pm 1.46$ & $4.49 \pm 1.44$ & $4.45 \pm 1.47$ \\
Total & $5.26 \pm 1.48$ & $5.11 \pm 1.47$ & $5.34 \pm 1.48$ \\
\hline
\end{tabular}

SD: Standard deviation; lnHF: log-transformed HF component values; Comparisons between men and women: $P$ value for mean, $t$-test.
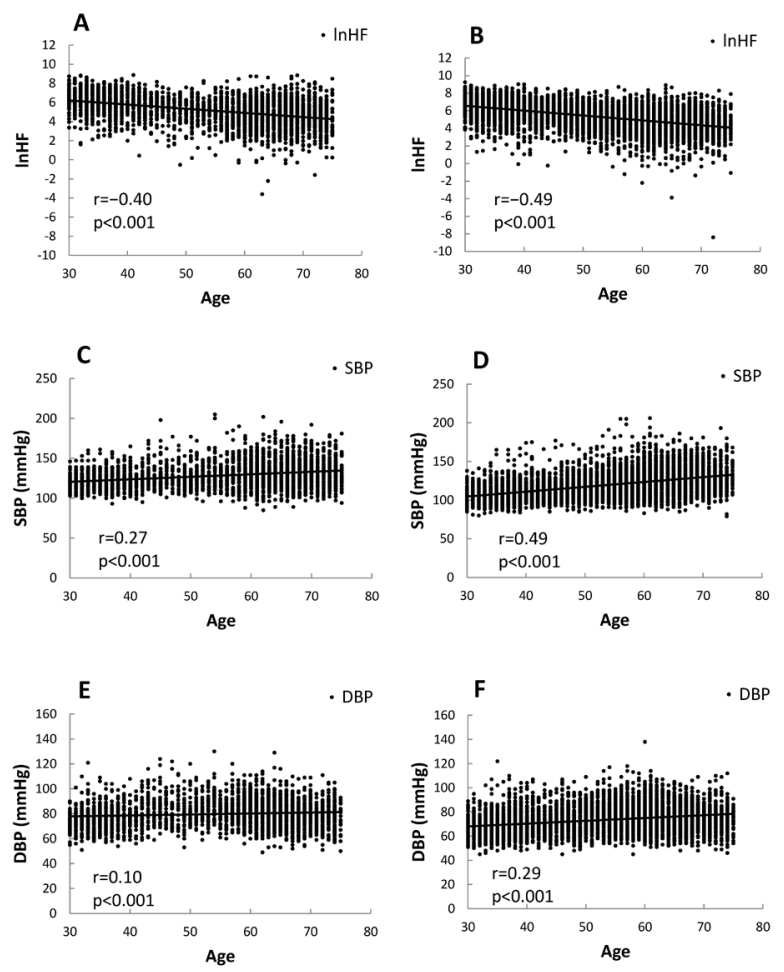

Figure 2. Scatter plots for log-transformed values of $\mathrm{HF}$ and age, systolic blood pressure, and diastolic blood pressure. (A): $\ln \mathrm{HF}$ vs. age (Men), (B): lnHF vs. age (Women), (C): SBP vs. age (Men), (D): SBP vs. age (Women), (E): DBP vs. age (Men), (F): DBP vs. age (Women). lnHF: log-transformed HF component values; SBP: systolic blood pressure; DBP: diastolic blood pressure.

results of trend test for the lnHF value of age groups showed a tendency for the lnHF value to decrease monotonically with increases in age group. This trend was observed in men, women, and in both sexes combined (each $P$ value for trend $<0.001$ ).

\subsection{Correlation between $\operatorname{lnHF}$ and age}

Figure 2 shows scatter plots and the Pearson correlation coefficient between age and $\ln \operatorname{HF}(r=-0.46)$. For comparison, we also show scatter plots and the Pearson correlation coefficients of age with SBP (0.42) and with DBP (0.22). In addition, the partial correlation coefficient excluding the effect of age for $\mathrm{lnHF}$ and SBP was $r=-0.29(P<0.001)$, indicating a weak negative correlation.

\subsection{Adjusted mean lnHF for each physical activity habit}

Figure 3 shows adjusted means of lnHF for each questionnaire item related to physical activity habits (adjusted for age) and the 95\% confidence intervals. The adjusted mean lnHF for the group with a greater degree of physical activity was higher than that for the lesser degree of physical activity.

\subsection{Impact of physical activity habits on HF component}

Table 3 shows multiple regression analysis results. We checked the variance inflation factor (VIF) for each of our models and found no multicollinearity problem (VIF $<3$ in all cases). Regression coefficients of physical activity habits were 0.133 [95\% confidence interval (95\%CI): 0.065-0.201] in Model A and 0.063 [0.008$0.118]$ in Model B. Coefficients for physical activity level in Model C were 0.079 [-0.017-0.176] (Sufficiently active) and 0.170 [0.062-0.278] (Highly active).

\section{Discussion}

Using 10-second ECG recordings for 9,135 general residents in a Japanese community, we found a moderate negative correlation between HF component values and age, in both sexes. The mean HF component value was higher in women than in men. HF component values increased with daily physical activity. This is one of the largest population studies that described the distributions of HF component values by age and sex, and found the positive relationship with daily physical activities.

We estimated and characterized the HF component values from 10-second ECG recordings for 9,135 adult residents in a Japanese community. Consistent with previous studies $(8,25,26)$, we confirmed lower HF component values with increased age. In addition, we clarified that women had relatively higher HF component values than men. Among those in their 30s to $50 \mathrm{~s}$, women had a higher HF component value than men, while those over 60 years of age showed only a small sex-dependent difference in HF component value. Thus, it is possible that age-related deterioration in parasympathetic function is more marked in women than in men. It is generally known that SBP is correlated with age (27). As shown in Figure 2, the strength of the 

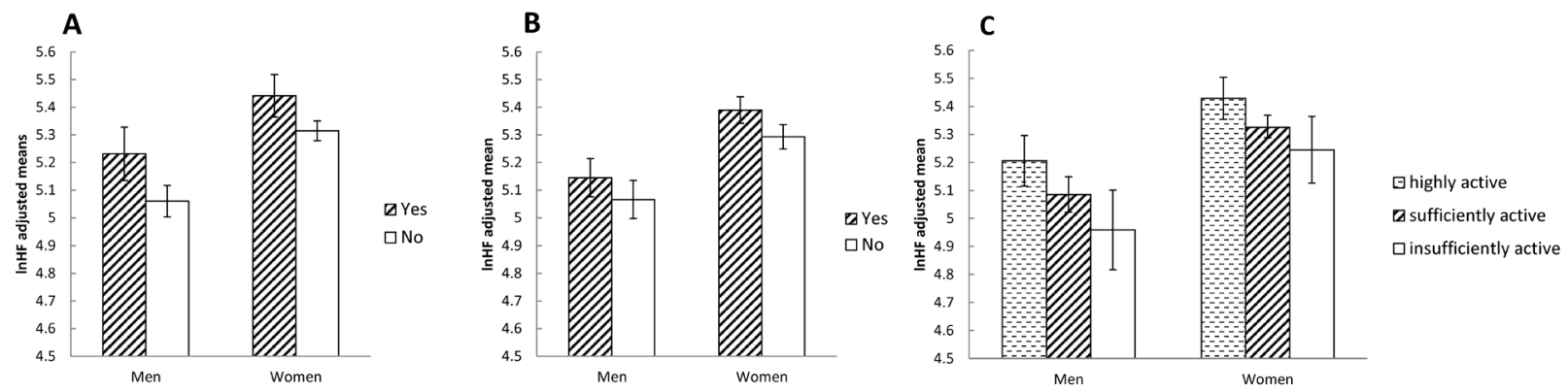

Figure 3. Adjusted mean InHF corresponding to each question related to physical activity (adjusted for age). (A): Are you in the habit of exercising to the point of light sweating for over 30 minutes per session, twice weekly, for over a year? 0: No, 1: Yes. (B): In your daily life do you walk or do an equivalent amount of physical activity for more than one hour a day? 0: No, 1: Yes. (C): How would you describe your physical activity level in your daily life? 1: Insufficiently active, 2: Sufficiently active, 3: Highly active. Error bar: $95 \%$ confidence interval. $P<$ 0.001 for all adjusted means.

Table 3. Regression model for InHF for each question related to physical activity

\begin{tabular}{|c|c|c|c|c|c|c|}
\hline \multirow{2}{*}{$\frac{\text { Model }}{\text { Model A }}$} & \multirow[t]{2}{*}{ Explanatory variable } & \multirow{2}{*}{$\begin{array}{l}\text { Coef. } \\
-0.051\end{array}$} & \multicolumn{2}{|c|}{$95 \% \mathrm{CI}$} & \multirow{2}{*}{$\begin{array}{l}P \text { value } \\
<0.001\end{array}$} & \multirow{2}{*}{$\frac{\operatorname{Adj} R^{2}}{0.225}$} \\
\hline & & & -0.055 & -0.049 & & \\
\hline & Men & & Refe & nce & & \\
\hline & Women & 0.031 & -0.028 & 0.090 & 0.299 & \\
\hline & BMI & -0.035 & -0.044 & -0.027 & $<0.001$ & \\
\hline & Exercise to the point of sweating: Yes & 0.133 & 0.065 & 0.201 & $<0.001$ & \\
\hline \multirow[t]{5}{*}{ Model B } & Age & -0.051 & -0.053 & -0.049 & $<0.001$ & 0.224 \\
\hline & Men & \multicolumn{4}{|c|}{ Reference } & \\
\hline & Women & 0.025 & -0.034 & 0.084 & 0.410 & \\
\hline & BMI & -0.035 & -0.043 & -0.026 & $<0.001$ & \\
\hline & Physical activity $>1 \mathrm{~h}$ : Yes & 0.063 & 0.008 & 0.118 & 0.024 & \\
\hline \multirow[t]{8}{*}{ Model C } & Age & -0.050 & -0.052 & -0.048 & $<0.001$ & 0.225 \\
\hline & Men & \multicolumn{4}{|c|}{ Reference } & \\
\hline & Women & 0.030 & -0.029 & 0.090 & 0.321 & \\
\hline & BMI & -0.034 & -0.043 & -0.026 & $<0.001$ & \\
\hline & \multicolumn{6}{|l|}{ Physical activity level } \\
\hline & Insufficiently active & \multicolumn{4}{|c|}{ Reference } & \\
\hline & Sufficiently active & 0.079 & -0.017 & 0.176 & 0.108 & \\
\hline & Highly active & 0.170 & 0.062 & 0.278 & 0.002 & \\
\hline
\end{tabular}

Coef.: regression coefficient, $95 \%$ CI: $95 \%$ confidence interval, Adj R²: adjusted R squired. Exercise to the point of sweating: Are you in the habit of exercising to the point of light sweating for over 30 minutes per session, twice weekly, for over a year? 0: No, 1: Yes. Physical activity >1 h: In your daily life do you walk or do an equivalent amount of physical activity for more than one hour a day? 0: No, 1: Yes. Physical activity level: How would you describe your physical activity level in your daily life? 1: Insufficiently active, 2: Sufficiently active, 3: Highly active.

negative correlation between age and HF component is quite comparable to the strength of the positive correlation between SBP and age. While autonomic nerve function has not been examined sufficiently in population settings, it may be one of the more important and easily assessed markers of aging. Kuo TB et al. pointed out that gender differences in parasympathetic function begin to decrease around age 50 (28).

The mean HF component values were greater in those who exercised regularly, relative to those who only performed regular light physical activity, even after adjusting for age. Similar findings pertaining to HF component were reported in a meta-analysis by Sandercock et al. (29). The HF component is an index of parasympathetic function. As the present study conducted spectral analyses of ECG data from 10-second measurements (which did not include the LF component), we were unable to determine whether parasympathetic dominance or sympathetic dominance was evident. However, as Aeschbacher et al. demonstrated, adopting a healthy lifestyle has an important effect on autonomic function (16), and we would surmise that maintaining good parasympathetic activity may be possible by performing regular physical activity. There are few studies that analyzed directly the relationship between daily physical activity habits and HRV in a large sample size. Several studies treated physical activity habits as theme in HRV analyses studies (30-34). Concerning the study on HRV and physical activity, communitybased studies using subjective physical activity data were at most about 5,000 subjects $(30,31)$. Studies using objective physical activity data were at most about 200 subjects (32-34). The present findings were based on one of the largest population studies that examined the relationships between HF component values and age, sex, and daily physical activities. 
Further empirical evidence is required in order to establish the utility of estimating autonomic nerve function from short ECG recordings to predict health outcomes. When HRV is measured in order to evaluate autonomic nervous function $(2,8)$, ECGs are conducted under strict experimental settings, and participants are prohibited from taking alcohol or caffeine for 24 hours prior to ECG measurement. Participants are also required to rest in the supine position for at least 15 minutes. Unfortunately, these processes make the measurement somewhat troublesome for screening examinees. At health checkups and clinical practice in Japan, the routine ECG recording time is approximately 10 seconds. In Japan, ECG is routinely used in daily clinical settings and for health checkups, and has been used in the National Survey on Circulatory Disorders to assess the health status among Japanese people (35). If remarkable parasympathetic dysfunction is found at this point, it may be possible to address early on any dysfunction in the autonomic nervous system. If the evaluation using 10-second ECG data proves to have the same or nearly similar accuracy as that for the conventional evaluation, this could be highly beneficial in outpatient therapy for cases such as patients with diabetic autonomic neuropathy or menopausal disorders in women. de Bruyne et al. found that an HRV analysis from 10-second ECG data was able to explain cardiac mortality among 5,272 elderly individuals (9). While there are many advantages of ECG in clinical practice, few studies have assessed the possibilities of using shorter duration ECG data to evaluate autonomic function. Further studies are needed to improve and disseminate knowledge on the potential clinical uses of HRV evaluation, particularly in terms of recording time and preconditioning.

The present study has some limitations. First, we were unable to consider the balance between the HF component and LF component when making conclusions on the relationship between physical activity habits and HF component. Second, it is known that HF component amplitude is reduced by increases in respiratory rate or decreased tidal volume, but we were only able to determine visually the presence or absence of breathing from the R-R interval fluctuation plot. Researchers' subjectivity must also be taken into account, so the presence/absence of breathing was not considered. However, respiratory influence might have occurred randomly among each age group, sex, and category of physical activity, thus there are little possibility of serious bias to the present findings. Moreover, we carefully investigated whether sleep or anxiety might be a confounding factor in examining the relationship between HF components and physical activity, but no remarkable relationship was found. Therefore, we focused simply on the relationship between HF components and physical activity. However, the present data concerning sleep and anxiety were collected only by self-administered questionnaire, thus, further studies are desirable with objective data on this issue.

In conclusion, we, using 10-second ECG data of community adult residents, found a moderate negative correlation between HF component values and age, in both sexes, although the mean HF component value was higher in women than in men. Increases in mean HF component values were also noted with increases in the level of daily physical activity. Maintaining the level of daily physical activity, especially to exercise regularly could keep the parasympathetic function high.

\section{Acknowledgements}

The authors would like to thank all participants, the Nagahama City Office, and the nonprofit organization Zeroji Club for their help in performing the Nagahama Study.

Funding: This study was supported by a research grant from the Takeda Science Foundation and university grants.

\section{References}

1. Akselrod S, Gordon D, Ubel FA, Shannon DC, Berger AC, Cohen RJ. Power spectrum analysis of heart rate fluctuation: a quantitative probe of beat-to-beat cardiovascular control. Science. 1981; 213:220-222.

2. Task Force of the European Society of Cardiology and the North American Society of Pacing and Electrophysiology. Heart rate variability: standards of measurement, physiological interpretation and clinical use. Circulation. 1996; 93:1043-1065.

3. Cooley JW, Tukey JW. An algorithm for the machine calculation of complex fourier series. Math Comput. 1965; 19:297-301

4. Ohtomo, N, Tanaka Y. New method of time series analysis and "MemCalc". In: A Recent Advance in TimeSeries Analysis by Maximum Entropy Method (Saito K, eds.). Hokkaido University Press, Sapporo, Japan, 1994; pp. 11-29.

5. Akaike H. Power spectrum estimation through autoregressive model fitting. Ann Inst Stat Math. 1969; 21:407-419.

6. Friederich HC, Schild S, Schellberg D, Quenter A, Bode C, Herzog W, Zipfel S. Cardiac parasympathetic regulation in obese women with binge eating disorder. Int J Obes (Lond). 2006; 30:534-542.

7. Liao D, Cai J, Rosamond WD, Barnes RW, Hutchinson RG, Whitsel EA, Rautaharju P, Heiss G. Cardiac autonomic function and incident coronary heart disease: a population-based case-cohort study. The ARIC Study. Atherosclerosis Risk in Communities Study. Am J Epidemiol. 1997; 145:696-706.

8. Hayano J. Shinpakuhendou ni yoru Jiritushinkeikinou Kaiseki (The autonomic function analysis by heart rate variability.). In: Junkankishikkan to Jiritushinkeikinou (Cardiovascular disease and Autonomic function.) (Inoue H, eds.), 2nd Edition. IGAKU-SHOIN Inc., Tokyo, Japan, 2001; pp. 75-77. (in Japanese)

9. de Bruyne MC, Kors JA, Hoes AW, Klootwijk P, Dekker 
JM, Hofman A, van Bemmel JH, Grobbee DE. Both decreased and increased heart rate variability on the standard 10-second electrocardiogram predict cardiac mortality in the elderly: the Rotterdam Study. Am J Epidemiol. 1999; 150:1282-1288.

10. Schroeder EB, Whitsel EA, Evans GW, Prineas RJ, Chambless LE, Heiss G. Repeatability of heart rate variability measures. J Electrocardiol. 2004; 37:163-172.

11. Nussinovitch U, Elishkevitz KP, Katz K, Nussinovitch M, Segev S, Volovitz B, Nussinovitch N. Reliability of Ultra-Short ECG Indices for Heart Rate Variability. Ann Noninvasive Electrocardiol. 2011; 16:117-122.

12. Blair SN, Kohl HW, Gordon NF, Paffenbarger RS, Jr. How much physical activity is good for health? Annu Rev Public Health. 1992; 13:99-126.

13. Paffenbarger RS, Jr., Hyde RT, Wing AL, Steinmetz CH. A natural history of athleticism and cardiovascular health. JAMA. 1984; 252:491-495.

14. Albinet CT, Boucard G, Bouquet CA, Audiffren M. Increased heart rate variability and executive performance after aerobic training in the elderly. Eur J Appl Physiol. 2010; 109:617-624.

15. Rezende Barbosa MP, Netto Junior J, Cassemiro BM, de Souza NM, Bernardo AF, da Silva AK, Pastre CM, Vanderlei LC. Impact of functional training on cardiac autonomic modulation, cardiopulmonary parameters and quality of life in healthy women. Clin Physiol Funct Imaging. 2016; 36:318-325.

16. Aeschbacher S, Bossard M, Ruperti Repilado FJ, Good N, Schoen T, Zimny M, Probst-Hensch NM, SchmidtTrucksass A, Risch M, Risch L, Conen D. Healthy lifestyle and heart rate variability in young adults. Eur J Prev Cardiol. 2016; 23:1037-1044.

17. Kluttig A, Schumann B, Swenne CA, Kors JA, Kuss O, Schmidt H, Werdan K, Haerting J, Greiser $\mathrm{KH}$. Association of health behaviour with heart rate variability: a population-based study. BMC Cardiovasc Disord. 2010; 10:58.

18. Britton A, Shipley M, Malik M, Hnatkova K, Hemingway $\mathrm{H}$, Marmot $\mathrm{M}$. Changes in heart rate and heart rate variability over time in middle-aged men and women in the general population (from the Whitehall II Cohort Study). Am J Cardiol. 2007; 100:524-527.

19. Takahashi N, Kuriyama A, Kanazawa H, Takahashi Y, Nakayama T. Validity of spectral analysis based on heart rate variability from 1-minute or less ECG recordings. Pacing Clin Electrophysiol. 2017; 40:1004-1009.

20. Tabara Y, Takahashi Y, Kohara K, Setoh K, Kawaguchi T, Terao C, Igase M, Yamada R, Kosugi S, Sekine A, Miki T, Nakayama T, Matsuda F. Association of longer QT interval with arterial waveform and lower pulse pressure amplification: the Nagahama Study. Am J Hypertens. 2013; 26:973-980.

21. Toyoshima H, Usami T, Chishaki A, Horibe H, Nichijunkyo shindenzu code 2005 no kaihatsu to sono keii (The development and background of the Nichijunkyo [The Japanese Society of Cardiovascular Disease Prevention] ECC code 2005 [compliant with 1982 version Minnesota code]). Nichijunyoboushi, 2005; 40:138-154. (in Japanese).

22. Prineas RJ, Crow RS, Zhang ZM. The Minnesota Code Manual of Electrocardiographic Findings, 2nd Edition. Springer-Verlag London Ltd, London, UK, 2010.

23. Sawada Y, Ohtomo N, Tanaka Y, Tanaka G, Yamakoshi K, Terachi S, Shimamoto K, Nakagawa M, Satoh S,
Kuroda S, Iimura O. New technique for time series analysis combining the maximum entropy method and non-linear least squares method: its value in heart rate variability analysis. Med Biol Eng Comput. 1997; $35: 318-322$.

24. Takusagawa M, Komori S, Umetani K, Ishihara T, Sawanobori T, Kohno I, Sano S, Yin D, Ijiri H, Tamura K. Alterations of autonomic nervous activity in recurrence of variant angina. Heart. 1999; 82:75-81.

25. Shannon DC, Carley DW, Benson H. Aging of modulation of heart rate. Am J Physiol. 1987; 253:H874-877.

26. Hrushesky WJ, Fader D, Schmitt O, Gilbertsen V. The respiratory sinus arrhythmia: a measure of cardiac age. Science. 1984; 224:1001-1004.

27. Franklin SS, Gustin Wt, Wong ND, Larson MG, Weber MA, Kannel WB, Levy D. Hemodynamic patterns of agerelated changes in blood pressure. The Framingham Heart Study. Circulation. 1997; 96:308-315.

28. Kuo TB, Lin T, Yang CC, Li CL, Chen CF, Chou P. Effect of aging on gender differences in neural control of heart rate. Am J Physiol. 1999; 277:H2233-2239.

29. Sandercock GR, Bromley PD, Brodie DA. Effects of exercise on heart rate variability: inferences from metaanalysis. Med Sci Sports Exerc. 2005; 37:433-439.

30. Jandackova VK, Scholes S, Britton A, Steptoe A. Are Changes in Heart Rate Variability in Middle-Aged and Older People Normative or Caused by Pathological Conditions? Findings From a Large Population-Based Longitudinal Cohort Study. J Am Heart Assoc. 2016; 5.

31. Soares-Miranda L, Sattelmair J, Chaves P, Duncan GE, Siscovick DS, Stein PK, Mozaffarian D. Physical activity and heart rate variability in older adults: the Cardiovascular Health Study. Circulation. 2014; 129:2100-2110.

32. Buchheit M, Simon C, Viola AU, Doutreleau S, Piquard F, Brandenberger G. Heart rate variability in sportive elderly: relationship with daily physical activity. Med Sci Sports Exerc. 2004; 36:601-605.

33. Hautala AJ, Karjalainen J, Kiviniemi AM, Kinnunen H, Makikallio TH, Huikuri HV, Tulppo MP. Physical activity and heart rate variability measured simultaneously during waking hours. Am J Physiol Heart Circ Physiol. 2010; 298:H874-880.

34. Soares-Miranda L, Sandercock G, Vale S, Santos R, Abreu S, Moreira C, Mota J. Metabolic syndrome, physical activity and cardiac autonomic function. Diabetes Metab Res Rev. 2012; 28:363-369.

35. Horibe H, Kasagi F, Kagaya M, Matsutani Y, Okayama A, Ueshima H. A nineteen-year cohort study on the relationship of electrocardiographic findings to all cause mortality among subjects in the national survey on circulatory disorders, NIPPON DATA80. J Epidemiol. 2005; 15:125-134.

Received May 8, 2020; Revised June 21, 2020; Accepted June 24, 2020.

*Address correspondence to:

Naomi Takahashi, Department of Health Informatics, Kyoto University School of Public Health, Yoshida Konoe-cho, Sakyo-ku, Kyoto 606-8501, Japan.

E-mail: ntakahashi@kuhp.kyoto-u.ac.jp

Released online in J-STAGE as advance publication July 4 2020. 